\title{
Elderly women described 5 phases of living with change after myocardial infarction
}

Sutherland B, Jensen L. Living with change: elderly women's perceptions of having a myocardial infarction. Qual Health Res 2000 Sep;10:661-76.

\section{QUESTION: What are elderly women's perceptions about having a myocardial infarction (MI)?}

Design

Exploratory design using a qualitative approach.

Setting

Edmonton, Alberta, Canada.

\section{Patients}

11 women (70-85 y) who were identified in 2 large tertiary care hospitals. Inclusion criteria were age $\geqslant 70$ years, a first MI, 8 weeks post-MI and had not received coronary artery bypass surgery, ability to speak and read English, ability to reflect on and verbalise perceptions and experiences, and residence within the city limits.

\section{Methods}

Women participated in unstructured interviews in their homes. Interviews were audiotaped and transcribed
For correspondence: Nursing, University of

Alberta, Edmonton, $A B$

T6G 2G3, Canada. Fax +17804922551 . verbatim. Content analysis was done and emerging themes were grouped into categories. Pattern coding was done to identify and explain emerging patterns. More comprehensive categories were developed for a more integrated understanding of events. Categories and themes were validated in telephone interviews with participants.

\section{Main findings}

Older women perceived the experience of having an MI as living with change. Throughout their experience, they faced challenges of being in control, managing uncertainty, making sense, being independent, and sheltering others. Having an MI was described as a 5 phase process: searching for a diagnosis, being hit with the reality, discovering the nature of the change, adjusting to the change, and moving on with the change. Not all women completed the process or moved through the phases sequentially. The process was cyclical for women who experienced a recurrence of symptoms. During the first phase, searching for a diagnosis, women became aware that a sudden change had occurred and they attempted to self diagnose and manage their symptoms. Women initially sheltered their family and friends by not seeking advice from them. In the second phase, being hit with the reality, the symptoms were verified, and some women reacted by detaching themselves from the events occurring in the emergency department, whereas others denied the nature of the change. Women reflected back on the diagnosis, their health histories, and related symptoms. During the third phase, discovering the nature of the change, women were admitted to hospital and adopted passive roles to cope with their diagnosis and the unfamiliar hospital environment. Family members sheltered them and monitored their progress. Although discharge teaching helped the women to translate the change, they arrived home with unanswered questions and feelings of uncertainty. In the fourth phase, adjusting to the change, women set limits as to how and when to make lifestyle changes. They depended on their families for support as they strove to regain independence. The fifth phase, moving on with the change, involved accepting the change on a personal level and getting on with life.

\section{Conclusions}

The central experience of older women who had a myocardial infarction (MI) was living with change. They described the strategies they used as being in control, managing uncertainty, making sense, being independent, and sheltering others through this process. Elderly women's abilities to live with the physical, cognitive, and emotional changes of having an MI affected their successful passage through this life event.
Morse JM, Johnson JL. The illness experience: dimensions of suffering. Newbury Park, CA: Sage Publications, 1991.

2 Schaefer KM. Celebrating life: a study of women who survived a major cardiac event. Pro1993. 